\title{
CONE-970980--1
}

\section{Diffuse scattering measurements of static atomic displacements in crystalline binary solid solutions}

\author{
G.E. Ice, C.J. Sparks, X. Jiang and L. Robertson \\ Oak Ridge National Laboratory, Oak Ridge TN 37831-6118
}

Diffuse $x$-ray scattering from crystalline solid solutions is sensitive to both local chemical order and local bond distances. In short-range ordered alloys, fluctuations of chemistry and bond distances break the long-range symmetry of the crystal within a local region and contribute to the total energy of the alloy. Recent use of tunable synchrotron radiation to change the $\mathrm{X}$-ray scattering contrast between elements has greatly advanced the measurement of bond distances between the three kinds of atom pairs found in crystalline binary alloys. The estimated standard deviation on these recovered static displacements approaches $\pm 0.001 \AA(0.0001 \mathrm{~nm})$ which is an order of magnitude more precise than obtained with EXAFS. In addition, both the radial and tangential displacements can be recovered to five near neighbors and beyond. These static displacement measurements provide new information which challenges the most advanced theoretical models of binary crystalline alloys.

\section{Introduction}

Although atomic size differences have long been recognized as critical to alloy design, experimental measurements of atomic size in crystalline alloys have been indirect or marginally precise. The recent availability of tunable $x$ rays from synchrotron sources now allows diffuse scattering measurements where the $x$-ray scattering contrast between the different elements in the sample can be enhanced or reduced. This has led to meaningful recovery of individual pair displacements to typically $\pm 0.001 \AA(0.0001 \mathrm{~nm}) .^{1-4}$ In a binary alloy of $\mathrm{A}$ and $\mathrm{B}$ atoms, the $\mathrm{AA}, \mathrm{AB}$, and $\mathrm{BB}$ average pair separation can be determined out to five or more atom shells (near neighbors). This new information presents a challenge to the theoretical community; theoretical models must allow for relaxation of the atoms away from the sites of the average lattice.

The long-range effect of substitutional alloying on the alloy lattice parameter is well characterized. The addition of large A atoms, to an alloy with small B atoms expands the lattice constant from the pure $B$ value. This is observed to be the case with a nearly linear response of the lattice constant to concentration throughout the solubility range and is often referred to as Vegard's law. ${ }^{5}$ Many models have been proposed to explain this linear relationship between the lattice parameter and elemental concentration. ${ }^{5-7}$ Though these models reproduce the nearly linear change in lattice parameter with concentration, actual measurements of the individual pair distances to test both models and theories have been almost non-existent or of questionable accuracy. 


\section{DISCLAMIER}

Portions of this document may be illegible in electronic image produets. Images are produced from the best available original document. 


\section{DISCLAIMER}

This report was prepared as an account of work sponsored by an agency of the United States Government. Neither the United States Government nor any agency thereof, nor any of their employees, make any warranty, express or implied, or assumes any legal liability or responsibility for the accuracy, completeness, or usefulness of any information, apparatus, product, or process disclosed, or represents that its use would not infringe privately owned rights. Reference herein to any specific commercial product, process, or service by trade name, trademark, manufacturer, or otherwise does not necessarily constitute or imply its endorsement, recommendation, or favoring by the United States Government or any agency thereof. The views and opinions of authors expressed herein do not necessarily state or reflect those of the United States Government or any agency thereof. 
The local effect of atomic size plays an important but still poorly understood role in alloy behavior. Atomic size disparity between solvent and solute is well-known to affect solubility and the physical/chemical properties of alloys. In a discussion of atomic size in alloys, Laves ${ }^{8}$ shows that the ratio of the atomic radii of the components affects their crystal symmetry. Solid solution strengthening or hardening increases with atomic size difference. ${ }^{9}$ Though Hume-Rothery ${ }^{10}$ recognized the role of atomic size differences on the structure and phase stability of alloys, the role and definition of atom size in metals with free electrons has remained elusive. Recent theoretical considerations stress the need to include static displacements in calculations of total energy. ${ }^{11}$

The most often quoted chemical displacements from an average lattice are obtained from extended $x$-ray absorption fine structure, EXAFS, measurements. EXAFS measurements are usually made in dilute alloys with the assumption that all nearest neighbor, nn, pairs to a solute atom are solvent atoms. ${ }^{12}$ EXAFS precision is typically $0.02 \AA(0.002 \mathrm{~nm})$ and at best $0.01 \AA(0.001 \mathrm{~nm})$ which for most alloys is marginal for measurement of the deviations of the atom pair spacings from the average long-range spacing. See reference 12 and those contained therein for a general discussion of EXAFS measurements and results.

Though diffuse scattering with $\mathrm{x}$ rays (neutrons and electrons) has been used since 1951 to provide information on the displacement of the atoms from the sites of the average lattice $e^{13,14}$, the practice of separating the individual pair displacements with selected $\mathrm{x}$-ray energies has only been developed recently. ${ }^{1-4}$ Details of the $\mathrm{x}$-ray measurements and data analysis to recover bond distances in alloys are found in references 4 and 15 . Here we focus on the reliability and implication of the average bond distances recovered from crystalline binary alloys with the so called "three $x$-ray energy" or " $3-\lambda$ " technique.

We present the information recovered with an emphasis on the static displacements of the atoms from the sites of the average lattice. Sufficient description is given so that the physical meaning of the displacements recovered from diffuse scattering data can be understood by the non-specialist. The discussion is confined to $x$-ray diffuse scattering measurements made on crystalline binary solid solution alloys where the average lattice is well defined by sharp (unbroadened) Bragg reflections. Defects such as stacking faults, high dislocation densities as from cold working, displacive transformations, incoherent precipitates and others defects which can broaden, split or produce new Bragg reflections are precluded as leaving an ill-defined average lattice. Such defects are treated elsewhere. ${ }^{16}$

For crystalline solid solutions the regular "d" spacing between crystallographic planes is maintained for hundreds, even thousands of planes as shown in Fig. 1; atoms are displaced out of these average planes in such a way as to maintain a regular "d" spacing. In order to define the static displacements to $\pm 0.001 \AA(0.0001 \mathrm{~nm})$ from this average lattice, the average planer spacing should vary by less than $\pm 0.001 \AA(0.0001 \mathrm{~nm})$. On differentiating Bragg's law, we obtain,

$$
\frac{\Delta d}{d}=\Delta \theta \cot \theta
$$

Here $\theta$ is the half angle between the incident and scattered $x$ ray. For a scattering angle of $2 \theta=40^{\circ}$, an average " $d$ " spacing of $2 \AA$ and an error in $\Delta d$ of $\pm 0.001 \AA(0.0001 \mathrm{~nm})$, then $\Delta \theta=0.01^{\circ}$ and the Bragg reflection would be broadened by $\Delta 2 \theta=0.02^{\circ}$. Substitutional crystalline solid solutions typically have sharper Bragg reflections than this $0.02^{\circ} \mathrm{FWHM}$. 


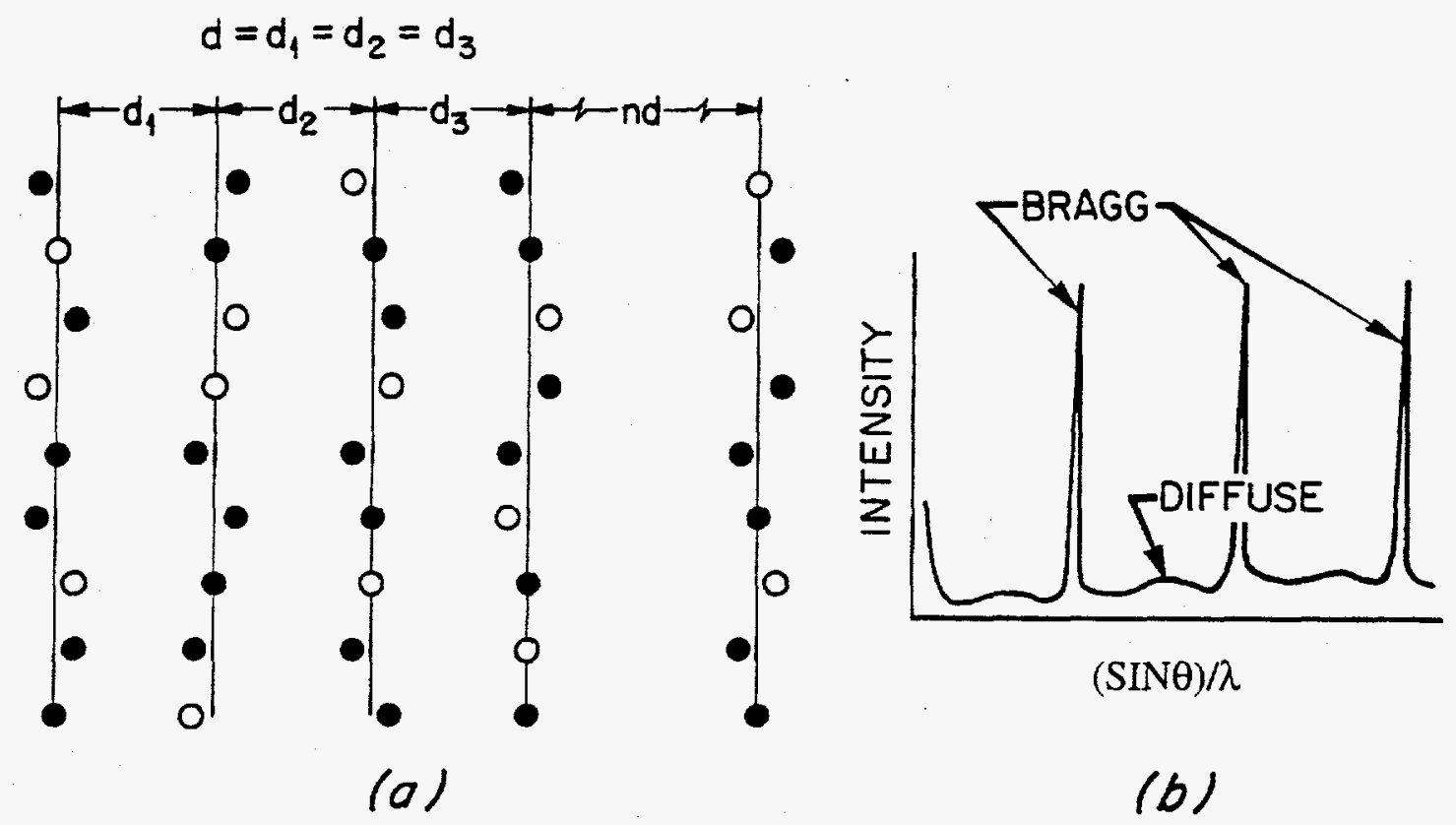

Figure 1. Displacement about the average lattice preserve the regular spacing between atomic planes such that $d=d_{1}=d_{2}=d_{3} \ldots$ The average lattice is obtained from (b) the positions of the sharp Bragg reflections. Information about short-range correlations among the atoms is contained in the diffusely distributed intensity between the Bragg peaks.

\section{Pair correlations from diffuse scattering}

\section{A. Diffraction theory of static displacement terms}

We give a brief overview of the kinematic diffraction theory in which the weak diffuse scattering can be approximated without extinction effects (first Born approximation). The purpose of this overview is to define the pair correlation parameters recovered from diffuse scattering measurements. Definitions of the atomic displacements are illustrated in Fig. 2 below.

The elastically scattered $x$-ray (neutron) intensity in electron units, eu, per atom from an ensemble of atoms is given by

$I(\mathbf{h})=\sum_{p} \sum_{q} f_{p} f_{q} e^{i 2 \pi \mathbf{h} \cdot\left(\mathbf{r}_{p}-\mathbf{r}_{q}\right)}$,

where $\mathrm{f}_{\mathrm{p}}$ and $\mathrm{f}_{\mathrm{g}}$ denote the complex $\mathrm{x}$-ray atomic scattering factor (or neutron scattering lengths); $p$ and $q$ designate the lattice sites, $\mathbf{r}_{\mathrm{p}}$ and $\mathbf{r}_{\mathrm{q}}$ are the position vectors for those sites, and $\mathbf{h}$ is the momentum transfer or reciprocal lattice vector $|\mathbf{h}|=(2 \sin \theta) / \lambda$. For crystalline solid solutions in which the Bragg reflections are sharp and the average lattice is welldefined, the atom positions can be represented by $\mathbf{r}=\mathbf{R}+\bar{\delta}$ where $\mathbf{R}$ is determined from the lattice constant and $\bar{\delta}$ is the displacement of the atom from that average lattice. The exponential term is written 


$$
e^{i 2 \pi \mathbf{h} \cdot\left[\left(\mathbf{R}_{p}-\mathbf{R}_{q}\right)+\left(\bar{\delta}_{p}-\bar{\delta}_{q}\right)\right]} \equiv e^{i 2 \pi \mathbf{h} \cdot\left(\mathbf{R}_{p}-\mathbf{R}_{q}\right)} e^{i 2 \pi \mathbf{h} \cdot\left(\bar{\delta}_{p}-\bar{\delta}_{q}\right)}
$$

and

$$
e^{i 2 \pi \mathrm{h} \cdot\left(\bar{\delta}_{p}-\vec{\delta}_{q}\right)} \equiv e^{i x}=1+i x-\frac{x^{2}}{2 !}-i \frac{x^{3}}{3 !}+\ldots+i \frac{x^{j}}{j !}+\ldots
$$

where $\mathrm{j}$ is an integer.

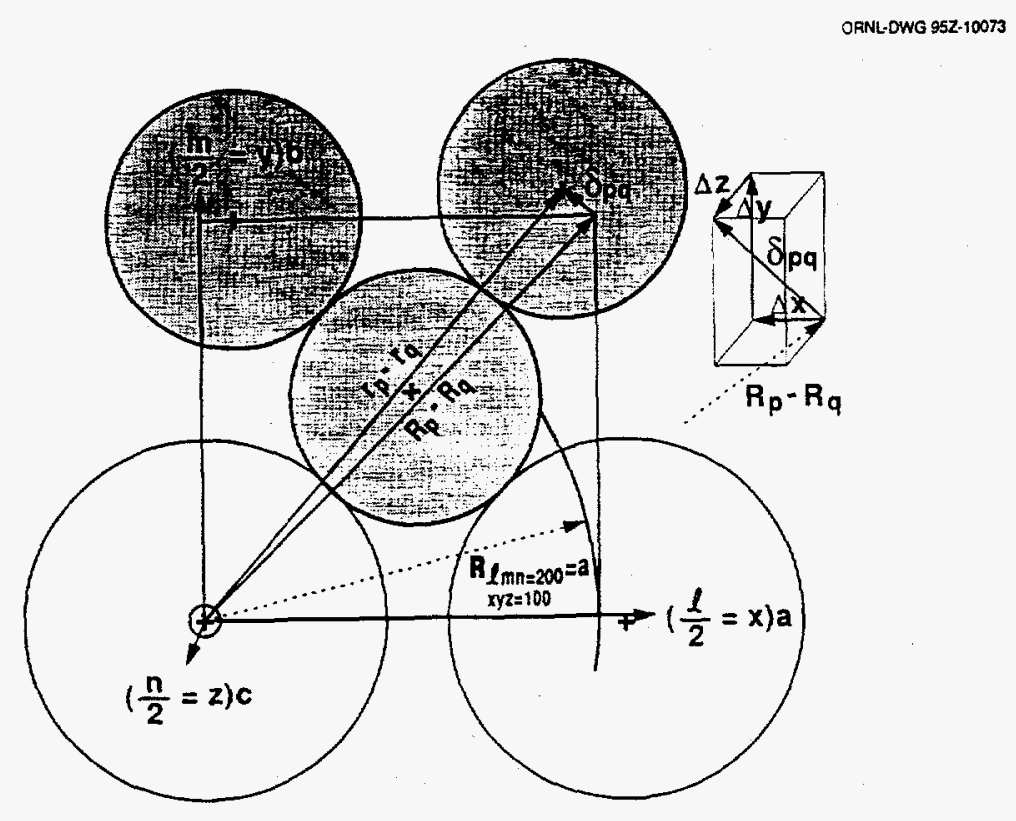

Figure 2. Schematic of a crystalline solid solution with atom pairs displaced by $\vec{\delta}_{p q}$ from the average lattice vector $\mathbf{R}_{\mathrm{p}}-\mathbf{R}_{\mathrm{q}}$. The + symbols represent the atom centers. The displacements are given in terms of their $l m n$ components designated $\Delta \mathbf{X}_{i m n}, \Delta \mathbf{Y}_{l m n}, \Delta \mathbf{Z}_{l m n}$ where $l=2 \mathrm{x}, m=2 \mathrm{y}$, and $n=2 z$ from an earlier convention for the designation of atom shells as integers.

This series expansion converges rapidly when h॰ $\bar{\delta}$ is sufficiently small. Upon substitution of Eq. (3b) into Eq. (2), the total intensity for a crystalline binary alloy can be written as follows:

$$
I_{T}=I_{F U N D}+I_{S R O}+I_{1 S D}+I_{j \geq 2}
$$

$I_{F U N D}+I_{S R O}$ represent the first term of the series expansion, $I_{1 S D}$ the second term (ix), and $I_{j \geq 2}$ the remainder. We follow the treatment of Warren and co-workers ${ }^{16}$ and write these terms for a cubic crystalline substitutional binary allow as

$$
\begin{aligned}
& I_{F U N D}=\left|c_{A} f_{A}+c_{B} f_{B}\right|^{2} \sum_{p q} \sum_{q} e^{2 \pi i h \cdot\left(R_{p}-R_{q}\right)} \\
& \frac{I_{S R O}}{N}=c_{A} c_{B}\left|f_{A}-f_{B}\right|^{2} \sum_{l m n} \alpha_{l m n} e^{-2 M \Phi_{l m n}} \cos \left[\pi\left(h_{1} l+h_{2} m+h_{3} n\right)\right]
\end{aligned}
$$




$$
\begin{aligned}
& \left.\frac{I_{1 S D}}{N}=-\frac{2 \pi}{a} c_{A} c_{B} \sum_{l m n} \sin \left[\pi\left(h_{1} l+h_{2} m+h_{3} n\right)\right]\right\}\left[\frac{c_{A}}{c_{B}}+\alpha_{l m n}\right] \\
& \times \operatorname{Re}\left[f_{A}\left(f_{A}-f_{B}\right)^{*}\right]\left[h_{1}\left\langle\Delta \mathbf{X}_{l m n}^{A A}\right\rangle+h_{2}\left\langle\Delta \mathbf{Y}_{l m n}^{A A}\right\rangle+h_{3}\left\langle\Delta \mathbf{Z}_{l m n}^{A A}\right\rangle\right] \\
& \left.-\left[\frac{c_{B}}{c_{A}}+\alpha_{l m n}\right] \operatorname{Re}\left[f_{B}\left(f_{A}-f_{B}\right)^{*}\right]\left[h_{1}\left\langle\Delta \mathbf{X}_{l m n}^{B B}\right\rangle+h_{2}\left\langle\Delta \mathbf{Y}_{l m n}^{B B}\right\rangle+h_{3}\left\langle\Delta \mathbf{Z}_{l m n}^{B B}\right\rangle\right]\right\} .
\end{aligned}
$$

Here $c_{A}$ is the $A$ atom fraction of $N$ atoms of a binary alloy, $c_{B}$ is the $B$ atom fraction, $f_{A}$ and $f_{B}$ are the respective complex atomic scattering factors and Re denotes the real part of a complex number. The lattice parameter of the cubic system is $a, \ell m n$ are the coordinates of the atom positions relative to the origin of the average lattice as shown in Fig. 2 , and $h_{1}$, $h_{2}$, and $h_{3}$ are the Cartesian coordinates of the momentum transfer vector $\mathbf{h}$ in reciprocal lattice units (r.l.u.). The Warren-Cowley short-range order coefficient $\alpha_{l m n}=1-p_{l m n}^{A B} / c_{B}$, where $p_{l m n}^{A B}$ is the conditional probability that after finding an $\mathrm{A}$ atom at $\ell m n$ there is a $\mathrm{B}$ atom at the origin. ${ }^{16}$ The displacements $\bar{\delta}_{p}-\bar{\delta}_{q} \equiv \bar{\delta}_{l m n} \equiv \Delta \mathbf{X}_{l m n}+\Delta \mathbf{Y}_{l m n}+\Delta \mathbf{Z}_{l m n}$ are illustrated in Fig. 2.

The average over all possible pairs that can be formed of the $\mathrm{X}$ components of the pair displacements for AA pairs with relative coordinates $\ell m n$ are given by $\left\langle\Delta \boldsymbol{X}_{l m n}^{A A}\right\rangle$ and similarly for $\mathrm{Y}$ and $\mathrm{Z}$ in the same units as the lattice constant. The average displacements for $\mathrm{AB}$ pairs $\left\langle\Delta \boldsymbol{X}_{l m n}^{A B}>\right.$ or their $\mathrm{Y}$ and $\mathrm{Z}$ components can be derived from the $\mathrm{AA}$ and $\mathrm{BB}$ displacements. By definition, the displacements are deviations from the average lattice; the weighted average of the displacements $\left\langle\bar{\delta}_{\ell m n}>\right.$ for all $\mathrm{AA}, \mathrm{AB}, \mathrm{BA}$, and $\mathrm{BB}$ pairs for any coordination shell is zero. Hence, we have that

$c_{A} p_{l m n}^{A A}\left\langle\bar{\delta}_{l m n}^{A A}\right\rangle+c_{A} p_{l m n}^{B A}\left\langle\bar{\delta}_{l m n}^{B A}\right\rangle+c_{B} p_{l m n}^{A B}\left\langle\bar{\delta}_{l m n}^{A B}\right\rangle+c_{B} p_{l m n}^{B B}\left\langle\bar{\delta}_{l m n}^{B B}\right\rangle=0$.

When rewritten in terms of $\alpha$ 's with $c_{A} p^{B A}=c_{B} p^{A B}$, and with $\left\langle\bar{\delta}_{l m n}^{A B}\right\rangle=\left\langle\bar{\delta}_{l m n}^{B A}\right\rangle$, we have that

$2\left(\alpha_{l m n}-1\right)\left\langle\Delta \boldsymbol{X}_{l m n}^{A B}\right\rangle=\left[\frac{c_{A}}{c_{B}}+\alpha_{l m n}\right]\left\langle\Delta X_{l m n}^{A A}\right\rangle+\left[\frac{c_{B}}{c_{A}}+\alpha_{l m n}\right]\left\langle\Delta X_{l m n}^{B B}\right\rangle$

Eq. $8 \mathrm{~b}$ insures that the interatomic vector averaged over all pairs in the crystal for each $l m n$ coordination shell is consistent with the average lattice long-range lattice parameter. ${ }^{13}$ For example $\mathbf{r}_{200}$ summed for all $\mathrm{AA}$. $\mathrm{AB}$, and $\mathrm{BB}<200>$ pairs and divided by the number of pairs must equal the crystal lattice constant (the average unit cell size, $a$ ). No assumption is made as to how the displacements are distributed about the average. This information is 
contained in the higher moments. Thus there are only two independent pair displacements for each shell of a cubic crystalline binary alloy. Equation (7) can be written in terms of any two of the three individual pair displacements according to Eq. (8b). We note that for widely separated atoms the first moment of the displacements goes to zero as they are equally likely to be displaced in a positive or negative direction relative to the atom at the origin. To evaluate the contribution from the term $\mathrm{I}_{\mathrm{j} \geq 2}$, we make the assumption that either the quadratic and high-order terms in this series expansion of the thermal and static displacements are the same for $\mathrm{AA}, \mathrm{AB}$ and $\mathrm{BB}$ atom pairs or that the different elements have nearly the same $x$-ray atomic scattering factors. ${ }^{1-t}$ This approximation is good for alloys with elements nearby in the periodic table as is the case of $\mathrm{Fe}-\mathrm{Ni}, \mathrm{Cr}-\mathrm{Fe}$ and $\mathrm{Cr}-\mathrm{Ni}$ alloys which have been studied to date. These alloys have similar masses (similar thermal motions), similar sizes (small static displacements) and similar number of electrons (similar $\mathrm{x}$-ray scattering factors). With this approximation, these remaining terms of the series expansion can be written as

$$
\begin{aligned}
\frac{I_{j \geq 2}}{N} & =\left|c_{A} f_{A}+c_{B} f_{B}\right|^{2} \frac{i^{j}}{j !} \sum_{l m n}\left\langle\left(\mathbf{h} \cdot \bar{\delta}_{l m n}\right)^{j}\right\rangle e^{2 \pi \mathrm{h} \bullet \boldsymbol{R}_{l m n}} \\
& +c_{A} c_{B}\left|f_{A}-f_{B}\right|^{2} \frac{i^{j}}{j !} \sum_{l m n} \alpha_{(l m n)}\left(\left(\mathbf{h} \cdot \bar{\delta}_{l m n}\right)^{j}\right) e^{2 \pi \mathrm{h} \bullet \boldsymbol{R}_{l m n}} .
\end{aligned}
$$

The first term of Eq. (9) weakens the fundamental Bragg reflections and distributes this intensity as temperature and static diffuse scattering. The second term of $E$. (9) weakens the superlattice reflections associated with long-range order or as is the case iere weakens the short-range order SRO diffuse maxima when there is only local order.

The second term of Eq. (9) which contains $\alpha_{\mathrm{Imn}}$ has been treated by Walker and Keating ${ }^{17}$ and is included as a thermal like factor $e^{-2 M \varphi_{l m n}}$ in Eq. (6). The effect of including this term on recovered $\alpha^{\prime}$ s is not more than $2 \%$ at room temperature. ${ }^{2,4}$ The first term of Eq. (9) includes quadratic and higher order thermal scattering and a smaller static diffuse scattering contribution. Typically the quadratic static scattering is less than the quadratic thermal scattering by a factor of three or more at room temperature even for alloys with large atomic size differences (e.g. AuCu$\left.)_{3}\right){ }^{18}$ Since this first term of Eq. (9) depends on $\left(c_{A} f_{A}+c_{B} f_{B}\right)^{2}$, its separation from $I_{S R O}$ and $I_{1 S D}$ (which depend on $f_{A}-f_{B}$ ) can be accomplished by choosing an $x$-ray energy such that $f_{A}-f_{B} \sim 0$. Thus the first term of Eq. (9) can be measured separately from $\mathrm{I}_{\mathrm{SRO}}+\mathrm{I}_{1 \mathrm{SD}}$ at one energy and scaled by $\left(\mathrm{c}_{\mathrm{A}} \mathrm{f}_{\mathrm{A}}+\mathrm{c}_{\mathrm{B}} \mathrm{f}_{\mathrm{B}}\right)^{2}$ to other energies. Measurements at three different $x$-ray energies and the recovery of the $\alpha^{\prime} s$ and $\delta^{\prime} s$ are described in references 1-4. A non-linear least-square fit to all three data sets is refined simultaneously with a program which includes the statistical errors on the input data. ${ }^{+}$

The precision with which the displacements can be recovered depends in part on how significant the term Eq. (7) is relative to the term Eq. (6), and on how large a contrast change can be effected in $\mathrm{f}_{A}\left(\mathrm{f}_{\mathrm{A}}-\mathrm{f}_{\mathrm{B}}\right)^{*}$ and $\mathrm{f}_{\mathrm{B}}\left(\mathrm{f}_{\mathrm{A}}-\mathrm{f}_{\mathrm{B}}\right)^{*}$; with $\mathrm{x}$ rays the scattering contrast varies with energy near the atomic absorption edges. ${ }^{+}$For atoms nearby in the periodic table the contrast can actually be reversed, and since $\left|f_{A}-f_{B}\right|$ is small, the displacement term containing $f_{A}\left(f_{A}-f_{B}\right)$ is large compared to the short-range order term containing $\left(f_{A}-f_{B}\right)^{2}$. 


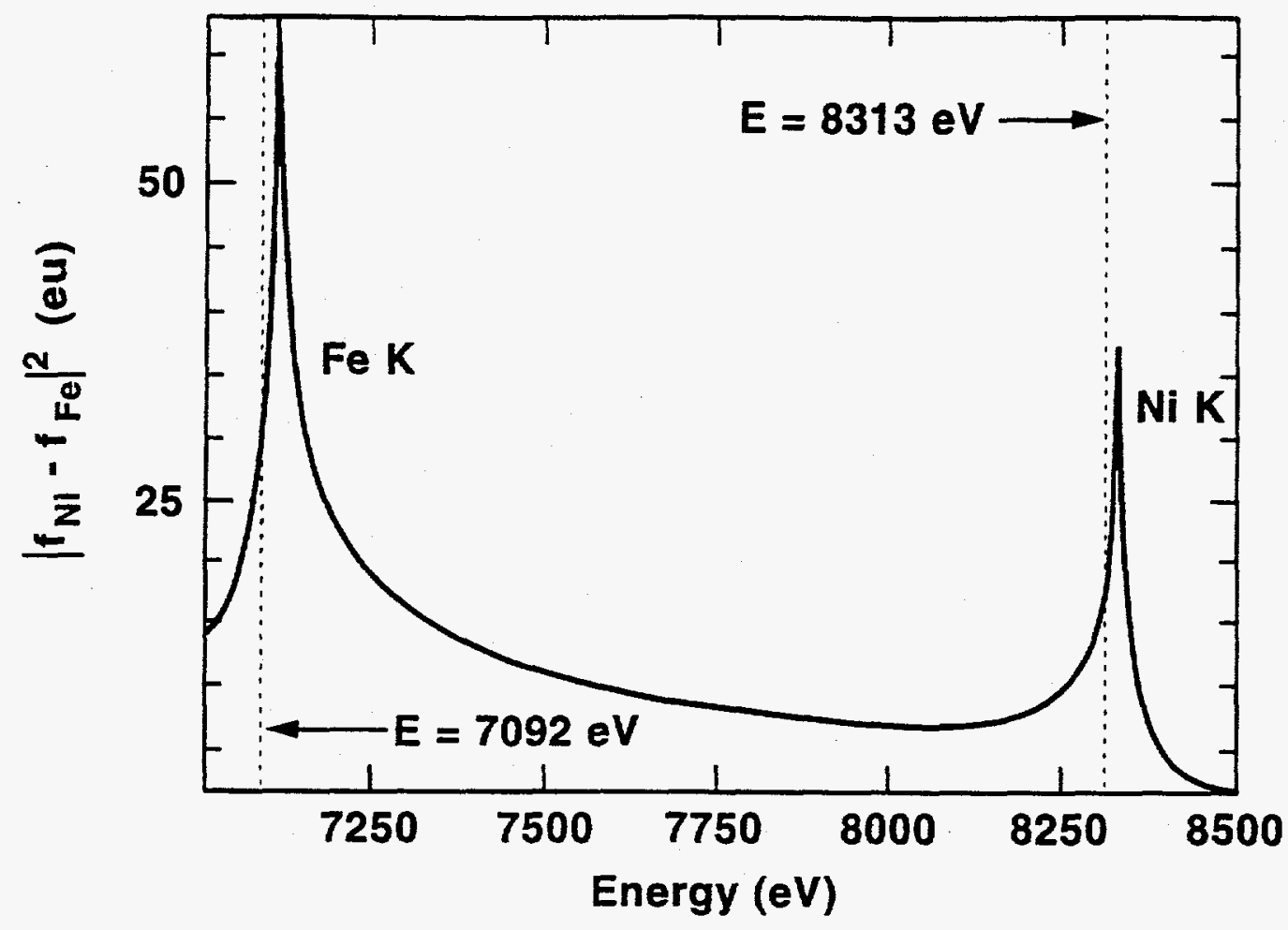

Figure 3. The variation in the Laue scattering term $\left|f_{\mathrm{Ni}}-f_{\mathrm{Fe}}\right|^{2}$ with $\mathrm{x}$-ray energy in the vicinity of the $\mathrm{Fe}$ and $\mathrm{Ni} \mathrm{K}$ absorption edges permits selection of $\mathrm{x}$-ray energies to change the contrast for recovery of the local chemical order and displacements among the individual atom pairs.

For Fe and $\mathrm{Ni}$ alloys, $\left(\mathrm{f}_{\mathrm{A}}-\mathrm{f}_{\mathrm{B}}\right)^{2}$ can be made to approach zero by proper choice of $\mathrm{X}$-ray energy (near $8500 \mathrm{eV}$ ) as shown in Fig. 3. The quadratic, higher order thermal and static displacement scattering along with the smearing functions of the experimental arrangement and possible multiple-scattering processes are approximately removed by subtracting the scaled null Laue data. Though the assumption is made here that the $\mathrm{A}$ and $\mathrm{B}$ atoms have similar quadratic and higher order displacements, possible errors introduced by this assumption are minimized by the choice of alloys with atoms of similar masses. The previous practice of calculating the thermal diffuse scattering (TDS) from force constants also assumes that constituent atoms have the same quadratic and higher order displacements. However, the use of force constants makes the harmonic approximation, and ignores both the high frequency acoustical and optical modes and the short-range correlations important to the scattering at the zone boundaries. All this is included in the measured null Laue scattering.

An example of the raw data measured for three different $x$-ray energies from an $\mathrm{Fe}_{46.5} \mathrm{Ni}_{53.5}$ alloy is shown in Fig. $4 .^{4}$ The solid line in Fig. 4 is the near null Laue measurement of the quadratic and higher order displacement terms which is removed from the other data sets to recover the $\alpha^{\prime} s$ and the $\delta^{\prime}$ s. Comparison of x-ray results with neutron diffuse scattering measurements which energy discriminate against thermal contributions give very similar $\alpha^{\prime} \mathrm{s}$ for $\mathrm{Fe}_{3} \mathrm{Ni}^{+}$ 


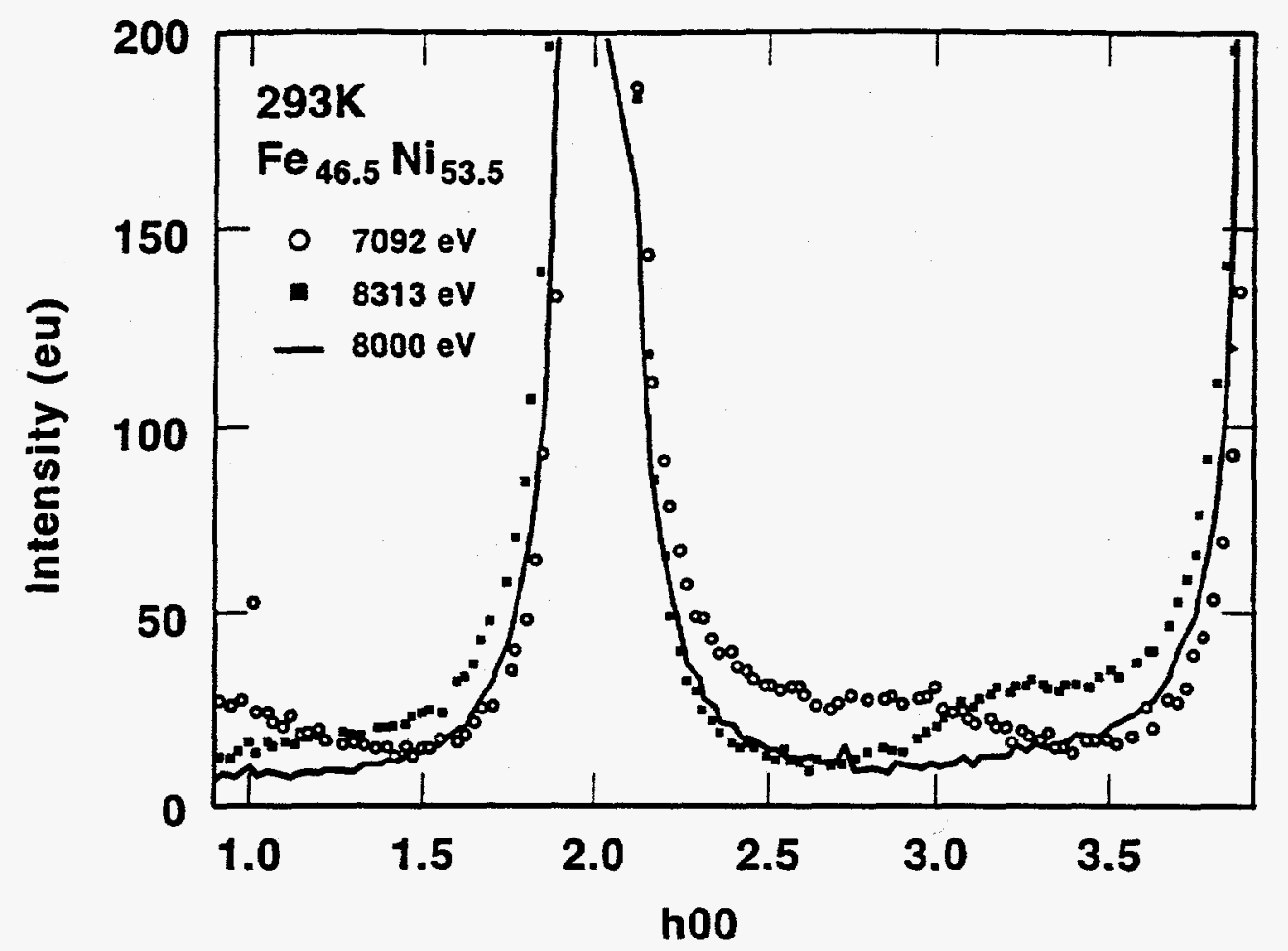

Figure 4. Total elastically scattered $\mathrm{x}$-ray intensity along the $<\mathrm{h} 00\rangle$ measured at $293 \mathrm{~K}$ for the three $\mathrm{x}$-ray energies listed. Note the shift in contrast for intensities measured with energies $20 \mathrm{eV}$ below the Fe K edge at $7092 \mathrm{eV}$ and the $\mathrm{Ni} \mathrm{K}$ edge at $8313 \mathrm{eV}$ which changes the sign of $\operatorname{Re}\left(\mathrm{f}_{\mathrm{N}_{\mathrm{i}}}-\mathrm{f}_{\mathrm{Fe}}\right)$. The outlying data point at the $\{100\}$ position is from harmonic energy contamination of the incident radiation and such points are removed before processing.

\section{Statistical and systematic errors}

The statistical uncertainties of the recovered parameters are estimated by propagating the standard deviation $\pm \sqrt{n}$ of the total number of counts $n$ for each data point through the non-linear least squares processing of the data. Systematic errors were determined by changing the values of input variables such as the $\mathrm{x}$-ray atomic scattering factors and reprocessing the data.

As input parameters were varied, the intensities were rescaled so that the $I_{S R O}$ values are everywhere positive and match values at the origin of reciprocal space measured by smallangle scattering. The integrated Laue scattering over a repeat volume in reciprocal space is also constrained to have an average value of $c_{A} c_{B}\left(f_{A}-f_{B}\right)^{2} ; \alpha_{000}=1$. These two constraints eliminate most of the systematic errors associated with converting the raw intensities into absolute units. ${ }^{19}$ The intensities measured at two different energies are adjusted to within $-1 \%$ on a relative scale and the intensity at the origin is matched to measured values. For these reasons, the standard deviations for $\alpha_{000}$ are estimated at $\sim 1 \%$.

Errors on the recovered $\alpha^{\prime} \mathrm{s}$ and $\Delta \mathrm{X}^{\prime}$ s arising from statistical and various possible systematic errors in the measurement and analysis of diffuse scattering data are given in Tables I and II for the $\mathrm{Fe}_{46.5} \mathrm{Ni}_{53.5}$ alloy. ${ }^{+.19 .21}$ Details of parameters used in the conversion to absolute intensity units are given in references 2 and 16 . A previous assessment of the 
systematic errors without the constraint of forcing $\alpha_{000}=1$ and without keeping the intensity at the origin and fundamentals a positive match to known values resulted in estimated errors being two to five times larger than those reported here..$^{20}$ Parameters necessary to the analysis of the data (other than well known physical constants) with our best estimate of their standard deviations and their contributing standard deviations to the $\alpha$ 's and $\Delta X$ 's are listed in Tables I and II. From a comparison of theoretical and measured values, we estimate a $0.2 \mathrm{eu}$ error on the real part of the $\mathrm{x}$-ray atomic scattering factors, a $1 \%$ error in the $\mathrm{P}_{0}$ calibration for converting the raw intensities to absolute unites (eu's), a 1 eu error in separating the inelastic resonant-Raman scattering, a 0-1 eu $\mathbf{h}$ dependent Compton scattering error ${ }^{23}$ and an error of \pm 0.3 at. \% in composition. ${ }^{+, 21}$ Systematic errors are larger than the statistical errors in this data.

Table I. Standard deviation of $\pm 1 \sigma$ for the uncertainties in the Warren-Cowley short-range order parameter $\alpha$ of $\mathrm{Fe}_{46.5} \mathrm{Ni}_{53,5}$ for statistical and possible systematic errors described in the text. Total error is shown in parenthesis. 0 indicates uncertainties less than 0.00005 .

\begin{tabular}{|l|c|c|c|c|c|c|c|}
\hline $\operatorname{lmn}$ & $\alpha_{1 \mathrm{mn}}\left(\sigma_{\text {Total }}\right)$ & $\sigma(\sqrt{n})$ & $\begin{array}{c}\sigma\left(\mathrm{f}^{\prime}\right) \\
\pm 0.2 \mathrm{eu}\end{array}$ & $\begin{array}{c}\sigma\left(\mathrm{p}_{0}\right) \\
\pm 1 \%\end{array}$ & $\begin{array}{c}\sigma(\mathrm{RRS}) \\
\pm 1 \mathrm{eu}\end{array}$ & $\sigma_{\text {Compton }}$ & $\begin{array}{c}\sigma\left(\mathrm{c}_{\mathrm{A}}\right) \\
\pm 0.3 \mathrm{at} \%\end{array}$ \\
\hline 000 & $1.0000(100)$ & 0.0024 & 0 & 0 & 0 & 0 & 0 \\
\hline 110 & $-0.0766(54)$ & 0.0018 & 0.0010 & 0.0048 & 0 & 0.0006 & 0.0011 \\
\hline 200 & $0.0646(28)$ & 0.0017 & 0.0003 & 0.0016 & 0.0008 & 0.0013 & 0.0003 \\
\hline 211 & $-0.0022(15)$ & 0.0014 & 0 & 0.0004 & 0.0001 & 0.0002 & 0.0001 \\
\hline 220 & $0.0037(14)$ & 0.0013 & 0.0002 & 0.0003 & 0.0003 & 0.0003 & 0.0001 \\
\hline 310 & $-0.0100(11)$ & 0.0011 & 0.0001 & 0.0002 & 0.0001 & 0.0001 & 0.0001 \\
\hline 222 & $0.0037(12)$ & 0.0011 & 0 & 0.0002 & 0.0002 & 0.0003 & 0 \\
\hline 321 & $-0.0032(19)$ & 0.0009 & 0 & 0.0001 & 0.0001 & 0.0001 & 0.0001 \\
\hline 400 & $0.0071(12)$ & 0.0011 & 0.0002 & 0.0001 & 0.0003 & 0.0004 & 0 \\
\hline 330 & $-0.0021(9)$ & 0.0008 & 0.0001 & 0 & 0.0003 & 0.0001 & 0 \\
\hline 411 & $0.0007(7)$ & 0.0007 & 0 & 0 & 0 & 0.0002 & 0 \\
\hline 420 & $0.0012(8)$ & 0.0007 & 0.0002 & 0 & 0.0004 & 0.0001 & 0 \\
\hline 332 & $-0.0007(7)$ & 0.0007 & 0 & 0 & 0 & 0.0001 & 0 \\
\hline
\end{tabular}

The asymmetric contribution of the first moment of the static displacements, $I_{1 S D}$ Eq. (7), to the diffuse intensity $\mathrm{I}_{\mathrm{SRO}}+\mathrm{I}_{1 \mathrm{SD}}$ for an $\mathrm{Fe}_{63.2} \mathrm{Ni}_{36.8}$ alloy is displayed in Fig. 5. ${ }^{24}$ Without static displacements the $\mathrm{I}_{\mathrm{SRO}}$ maxima would occur at the 100 and 300 superlattice positions. The static atomic displacements for the alloy are similar to those given in table II. Such large distortions of the short-range order diffuse scattering caused by displacements of $<0.02 \AA(0.002 \mathrm{~nm})$ emphasizes the sensitivity of this technique. On changing $\mathrm{x}$-ray energy from 7.092 to $8.313 \mathrm{keV}, f_{\mathrm{Ni}}$ becomes smaller than $f_{\mathrm{Fe}}$. Figure 5 displays a reversal in the shift of the position of the diffuse scattering maxima. Our choice of these two $\mathrm{X}$-ray energies for the $3 \lambda$ method are to emphasize this contrast and then to choose a third nearest the null Laue energy for removal of the TDS. The total estimated standard deviation on the values of the $\alpha^{\prime}$ s and in particular the $\Delta X^{\prime}$ s give unprecedented precision for the displacements with errors $\pm 0.003 \hat{\AA}$ and less. 
Table II. Standard deviation of $\pm 1 \sigma$ of $x, y$ and $z$ components of the pair $\mathrm{Fe}-\mathrm{Fe}$ displacements $\bar{\delta}_{\text {Fe-Fe }}$ for the various atom pairs of $\mathrm{Fe}_{46.5} \mathrm{Ni}_{53.5}$ for statistical and possible systematic errors described in the text. Total error is shown in parenthesis. 0 indicates uncertainties less than $0.00005 \AA$.

\begin{tabular}{|l|c|c|c|c|c|c|c|}
\hline $\operatorname{lmn}$ & $\begin{array}{c}\Delta \mathrm{X}\left(\sigma_{\text {Total }}\right) \\
(\mathrm{A})\end{array}$ & $\sigma(\sqrt{n})$ & $\begin{array}{c}\sigma\left(\mathrm{f}^{\prime}\right) \\
\pm 0.2 \mathrm{eu}\end{array}$ & $\begin{array}{c}\sigma\left(\mathrm{p}_{0}\right) \\
\pm 1 \%\end{array}$ & $\begin{array}{c}\sigma(\mathrm{RRS}) \\
\pm 1 \mathrm{eu}\end{array}$ & $\sigma_{\text {Compton }}$ & $\begin{array}{c}\sigma\left(\mathrm{c}_{\mathrm{A}}\right) \\
\pm 0.3 \text { at. \% }\end{array}$ \\
\hline 110 & $0.0211(25)$ & 0.0002 & 0.0023 & 0.0007 & 0.0002 & 0.0004 & 0.0004 \\
\hline 200 & $-0.0228(14)$ & 0.0004 & 0.0010 & 0.0007 & 0.0002 & 0.0004 & 0.0002 \\
\hline 211 & $0.0005(2)$ & 0.0002 & 0 & 0.0001 & 0.0001 & 0 & 0 \\
\hline 121 & $0.0014(4)$ & 0.0001 & 0.0003 & 0.0001 & 0.0002 & 0 & 0 \\
\hline 220 & $0.0030(7)$ & 0.0002 & 0.0006 & 0.0001 & 0.0003 & 0.0001 & 0 \\
\hline 310 & $0.0022(3)$ & 0.0002 & 0.0001 & 0.0001 & 0.0002 & 0.0001 & 0 \\
\hline 130 & $0.0009(2)$ & 0.0002 & 0.0001 & 0 & 0.0001 & 0 & 0 \\
\hline 222 & $0.0003(3)$ & 0.0002 & 0.0002 & 0 & 0.0001 & 0 & 0 \\
\hline 321 & $0.0011(2)$ & 0.0001 & 0.0001 & 0 & 0.0002 & 0 & 0 \\
\hline 231 & $0.0001(1)$ & 0.0001 & 0 & 0 & 0.0001 & 0 & 0 \\
\hline 123 & $0.0008(4)$ & 0.0001 & 0.0001 & 0 & 0 & 0 & 0 \\
\hline 400 & $-0.0019(6)$ & 0.0004 & 0.0002 & 0.0001 & 0.0003 & 0.0001 & 0 \\
\hline 330 & $0.0011(4)$ & 0.0002 & 0.0001 & 0 & 0.0003 & 0 & 0 \\
\hline 411 & $-0.0008(3)$ & 0.0002 & 0.0002 & 0 & 0.0002 & 0 & 0 \\
\hline 141 & $-0.0001(2)$ & 0.0001 & 0.0001 & 0 & 0.0001 & 0 & 0 \\
\hline
\end{tabular}

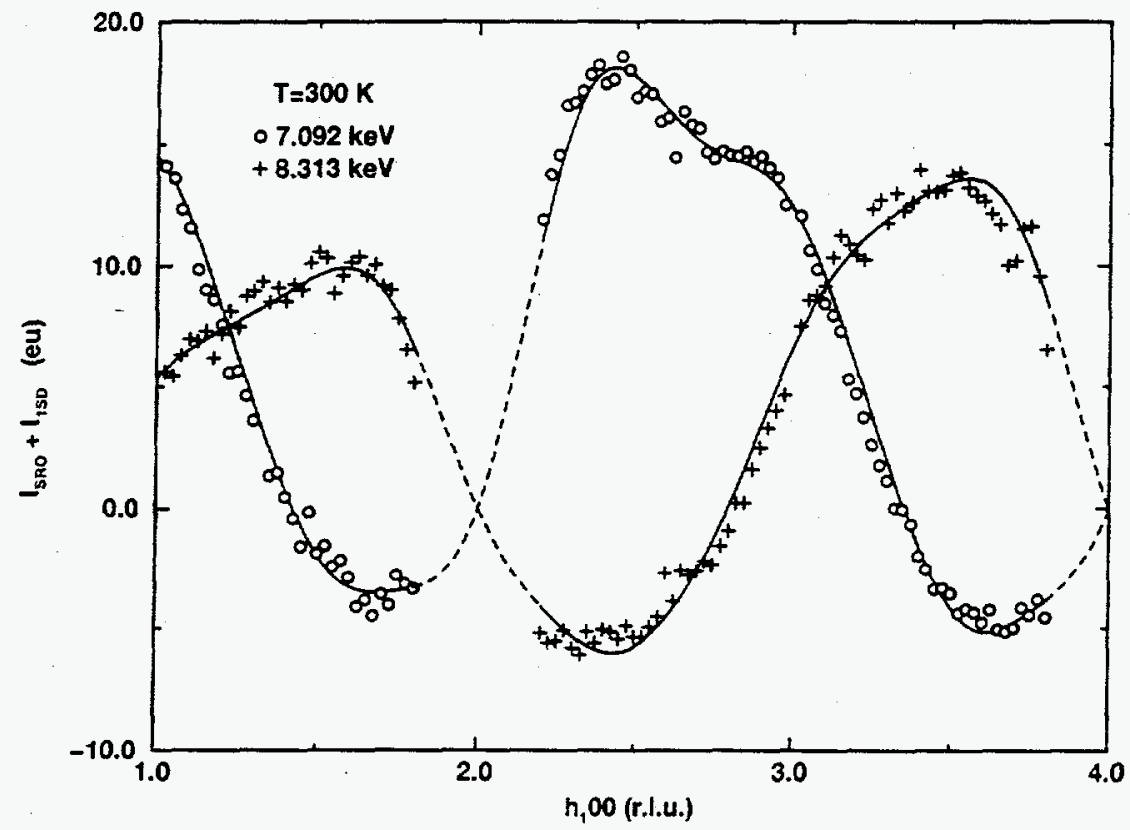

Figure 5. Diffusely scattered $\mathrm{x}$-ray intensity from an $\mathrm{Fe}_{63}{ }_{2} \mathrm{Ni}_{36.8}$ Invar alloy associated with the chemical order $I_{S R O}$ and the first moment of the static displacements $I_{\text {ISD }}$ along the $\left[h_{1} 00\right]$ direction. A major intensity change is affected by the choice of two different $x$-ray energies. The solid lines calculated from the $\alpha^{\prime}$ s and $\delta^{\prime}$ srecovered from the $3 \lambda$ data sets closely fit the observed data given by $o$ and + . The dashed lines are calculated intensity through the fundamental reflections. (From reference 24). 


\section{$\underline{\text { IV. Static Atomic Displacements }}$}

\section{A. Meaning of recovered static displacements}

Since the $\mathrm{x}$-ray beam is about a millimeter in diameter and penetrates a few microns into the sample, $\sim 10^{17}$ atoms contribute to the diffraction pattern with $\sim 10^{18}$ first neighbor pairs. From Eq. (3a)

$\mathbf{r}_{p}-\mathbf{r}_{q}=\left(\mathbf{R}_{p}-\mathbf{R}_{q}\right)+\left(\bar{\delta}_{p}-\bar{\delta}_{q}\right)$

and as we can move the frame of reference so that its origin always resides on one of the atoms of the pair such that $\mathbf{r}_{\mathbf{p}} \equiv \mathbf{r}_{0} \equiv 0, \mathbf{R}_{\mathrm{p}} \equiv \mathbf{R}_{0} \equiv 0$ and $\delta_{\mathrm{p}} \equiv \delta_{0} \equiv 0$, then

$\mathbf{r}_{p}-\mathbf{r}_{q}=\mathbf{r}_{0}-\mathbf{r}_{q}=\mathbf{r}_{0}-\mathbf{r}_{l m n}=-\mathbf{r}_{l m n}$

and with the atom pair identified by ij

$\mathbf{r}_{l m n}^{i j}=\mathbf{R}_{l m n}+\delta_{l m n}^{i j}$

where $\mathbf{R}_{\mathrm{Imn}}$ is independent of the kinds of atom pairs since it is defined by the average lattice, i.e., Bragg reflection positions. The average value of the measured $\mathbf{r}_{\mathrm{lm} n}$ for all the $\mathrm{N}$ pairs contributing to the measured intensity is

$\left\langle\mathbf{r}_{l m n}^{i j}\right\rangle=\frac{1}{N^{i j}} \sum_{i j}\left\langle\mathbf{R}_{l m n}+\delta_{l m n}^{i j}\right\rangle=\mathbf{R}_{l m n}+\left\langle\delta_{l m n}^{i j}\right\rangle$

Here $\left\langle\delta_{l m n}^{j j}\right\rangle$ is the variable recovered from the diffuse scattering. As shown in Eq. (7), we can recover the rectangular coordinates of the average displacement vector,

$\left\langle\bar{\delta}_{l m n}^{i j}\right\rangle \equiv\left\langle\Delta \mathbf{X}_{l m n}^{i j}\right\rangle+\left\langle\Delta \mathbf{Y}_{l m n}^{i j}\right\rangle+\left\langle\Delta \mathbf{Z}_{l m n}^{i j}\right\rangle$

For cubic systems when the atom has less than 24 neighboring atoms in a coordination shell (permutations and combinations of $\pm l, \pm m, \pm n$ ), $\left\langle\bar{\delta}_{l m n}^{i j}\right\rangle$ must be parallel to the interatomic vector $\mathbf{R}_{l m n}$. This maintains the statistically observed long range cubic symmetry even though on a local scale this symmetry is broken. For $l m n$ multiplicities $\geq 24$, the displacements on the average need not be parallel to the average interatomic vector $\mathbf{R}_{l m n}$ to preserve cubic symmetry ${ }^{22}$.

Measurements of diffuse scattering from single crystals provides the vector components of the atomic displacements $\langle\Delta X\rangle,\langle\Delta Y\rangle$, and $\langle\Delta Z\rangle$; whereas, the spherical average obtained from EXAFS and $x$-ray measurements on amorphous materials and crystalline powders gives only the magnitude of the radial displacements. Thus, diffuse $x-$ ray scattering provides new information about the vector displacements associated with near neighbor chemistry. 


\section{B. Discussion of measured displacements}

Measured displacements such as presented in Table II provide unique insight into how atoms move off their lattice sites when local symmetry is broken. Local symmetry is broken when a multicomponent crystalline material is above the ordering temperature (with less than perfect long-range order) and/or off stoichiometry. With perfect long-range order the atoms are constrained to lie precisely on the sites of the average lattice by balanced forces. In alloys where the local symmetry is broken, we gain new insights into the chemically distinct bonding, including the interatomic bond distances and whether the displacements have both radial and tangential components. With reference to Fig. 2, the displacement for the [110] nearest neighbor atoms is on average radial with a magnitude given by $k \bar{\delta}_{110}>|=\sqrt{2}| \Delta \mathbf{X}_{110} \mid$.

We note that the Fe-Fe first neighbor pair distances given in Table II are 0.021 (3) $\AA$ $\mathrm{x} \sqrt{2}=0.030(4) \AA$ further apart then the average lattice and that second neighbor pairs are closer by (-) $0.023(1) \AA$. Average bond distances along the interatomic vector between nearest neighbor pairs for this fcc lattice are obtained by adding the $\sqrt{2}\left|\Delta \mathbf{X}_{110}\right|$ to the average interatomic vector $\mathbf{R}_{110}$ as defined in Fig. 2 . $\mid \mathbf{R}_{110}$ is just the cubic lattice constant a times $1 / \sqrt{2}$. From the construction shown in Fig. 6 , it follows that the vector distance between a pair of atoms, $\mathbf{r}_{l m n}^{i j}$, has radial and tangential displacement components with magnitudes given by,

$$
\left|\bar{\delta}_{l m n}^{i j}\right|_{\|}=\frac{\bar{\delta}_{l m n}^{i j} \cdot \mathbf{R}_{l m n}}{\left|\mathbf{R}_{l m n}\right|}
$$

and

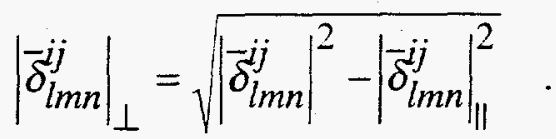

The radial (II) and tangential ( $\perp$ ) components of the displacements recovered from diffuse scattering measurements on single crystals are shown in Fig. 6. 


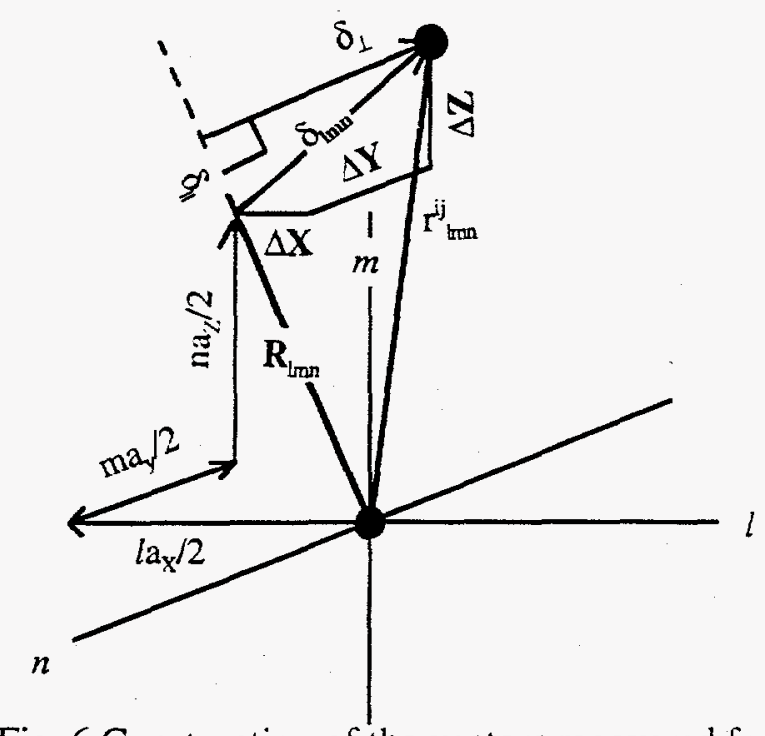

Fig. 6 Construction of the vectors recovered from diffuse scattering measurements on single crystals. $\mathrm{R}_{\mathrm{lmn}}$ is obtained from the lattice parameter $a$, and the average components of the displacement $\delta_{l m n}^{i j}$ are recovered from measurements of the diffuse scattering.

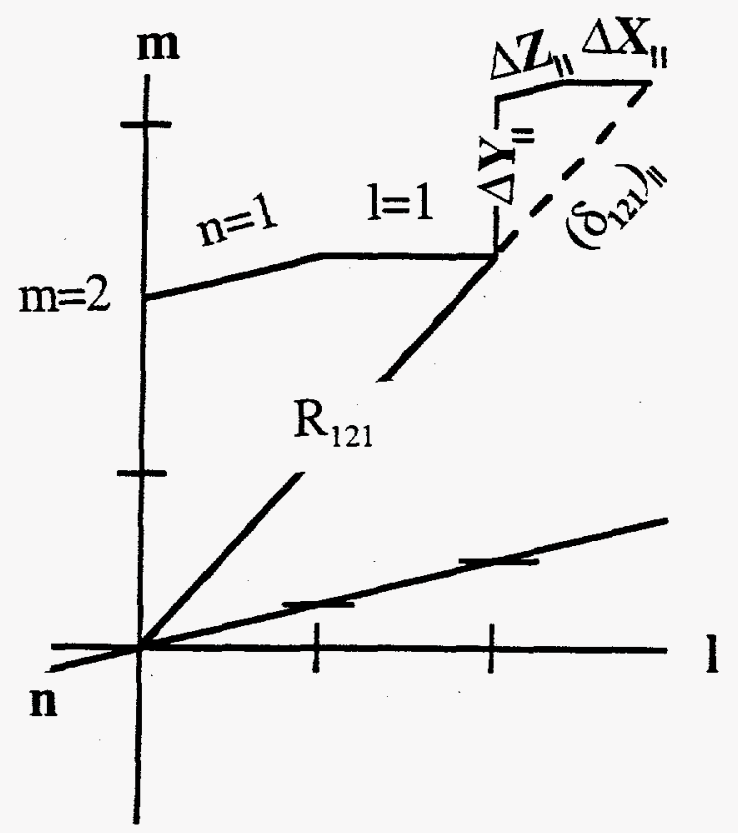

Fig. 7 Radial displacements (parallel to the interatomic vector $\mathrm{R}_{i m n}$ ) between the atom pairs require that the relative magnitudes of the displacement components be in the same proportion as the average lattice vector: $\Delta \mathrm{X}: \Delta \mathrm{Y}: \Delta \mathrm{Z}=l: m: n$. As shown for $l m n=211$, a radial displacement requires $|\Delta \mathrm{X}|=2|\Delta \mathrm{Y}|$ and $|\Delta \mathrm{X}|=2|\Delta \mathrm{Z}|$. For $l m n=121,|\Delta \mathrm{X}|=|\Delta \mathrm{Y}| / 2$ and $|\Delta \mathbf{X}|=|\Delta \mathbf{Z}|$. For a cubic lattice we can interchange $l, m$, and $n$ and similarly $\Delta X ; \Delta Y$; and $\Delta Z$. Thus there is only one value $\Delta X$ for $l m n$ multiplicities $<24$, i.e., $110,200,222$, etc., two values for $\Delta \mathrm{X}$ when $l m n$ has multiplicities equal to $24(l \neq m$ and $l=m, n)$ and three values for $\Delta \mathrm{X}$ with multiplicities equal to 48 . 
As the $\mathrm{Fe}_{46.5} \mathrm{Ni}_{53.5}$ alloy is cubic (face centered), the $\Delta \mathrm{Y}$ and $\Delta \mathrm{Z}$ dispiacements are derived from the $\Delta X$ 's given in Table II by permutation of the indices. (Henceforth, we drop the $>$ on the displacements for simplicity.) For example, $\Delta \mathrm{X}_{321}$ has the identical value as $\Delta \mathrm{Y}_{231}$ and as $\Delta \mathrm{Z}_{123}$, and $\Delta \mathrm{X}_{321}=\Delta \mathrm{X}_{312}=\Delta \mathrm{Y}_{231}=\Delta \mathrm{Y}_{132}=\Delta \mathrm{Z}_{123}=\Delta \mathrm{Z}_{213}$. In addition $\Delta \mathrm{X}_{321}=-\Delta \mathrm{X}_{321}^{-}$and similarly for the other combinations as illustrated in Fig. $7^{22}$. The nearest atom pairs which could exhibit non-radial components are those in the third neighboring shell, $\operatorname{lmn}=211$.

If the displacements between atom pairs is on the average along their interatomic vector, then $\Delta \mathrm{X}_{211}=2 \Delta \mathrm{X}_{121}$. For the Fe-Fe pair displacements given in Table II, $\Delta \mathrm{X}_{211}=$ $0.0005(2) \AA$ and $2 \Delta \mathrm{X}_{121}=0.0028(8) \AA$, thus the (211) Fe-Fe pair displacements have a significant tangential component. From Eqs. (15a) and (15b), the magnitude of the displacement between (211) Fe-Fe pairs along the radial direction $\left|\delta_{211}^{F e}\right|_{\|}$is 0.0016 (7) $\AA$ and $\left|\delta_{211}^{F e-F e}\right|_{\perp}$ tangential is $0.0013(7) \AA$. Thus the (211) Fe-Fe neighbors have a similar radial and tangential component to their displacements. For the (310) Fe-Fe pair displacements, $\Delta \mathrm{X}_{310} \sim 3 \Delta \mathrm{X}_{130}$ within the total estimated error, and on the average (310) displacements are predominantly radial. These measured displacements provide new information not obtained in other ways about the local atomic arrangements in crystalline solid solutions.

Only a few crystalline binary alloys have had their individual pair displacements measured with this $3 \lambda$ technique. They include $\mathrm{Fe}_{22.5} \mathrm{Ni}_{77.5}{ }^{1,4}, \mathrm{Fe}_{46.5} \mathrm{Ni}_{53.5}{ }^{4}, \mathrm{Cr}_{47} \mathrm{Fe}_{53}{ }^{2}$ and $\mathrm{Cr}_{20} \mathrm{Ni}_{30}{ }^{3}$. These results are summarized in Fig. 8 where the $\Delta \mathrm{X}$ static displacements are plotted as a function of the radial distance $\frac{1}{2} \sqrt{l^{2}+m^{2}+n^{2}}$. When there is more than one value for $\Delta \mathrm{X}$, the plots show the various values. Most striking is the observation that for the three ordering alloys the near neighbor $\mathrm{Fe}-\mathrm{Ni}$ and $\mathrm{Cr}$-Ni bond distances are the smallest of the three possible pairs (Fig. 8A, 8B, and 8C). However, for the clustering $\mathrm{Cr}_{47} \mathrm{Fe}_{53}$ alloy the $\mathrm{Cr}-\mathrm{Cr} \mathrm{nn}$ bond distances are closest and the $\mathrm{Cr}-\mathrm{Fe}$ furthest apart. More details including the short-range order parameters $\alpha$ and numerical values of the displacements for each shell are given in the original papers. These pair displacement observations provide a more rigid test of theoretical predictions than variations of the average lattice parameter with concentration ${ }^{25,26}$. 

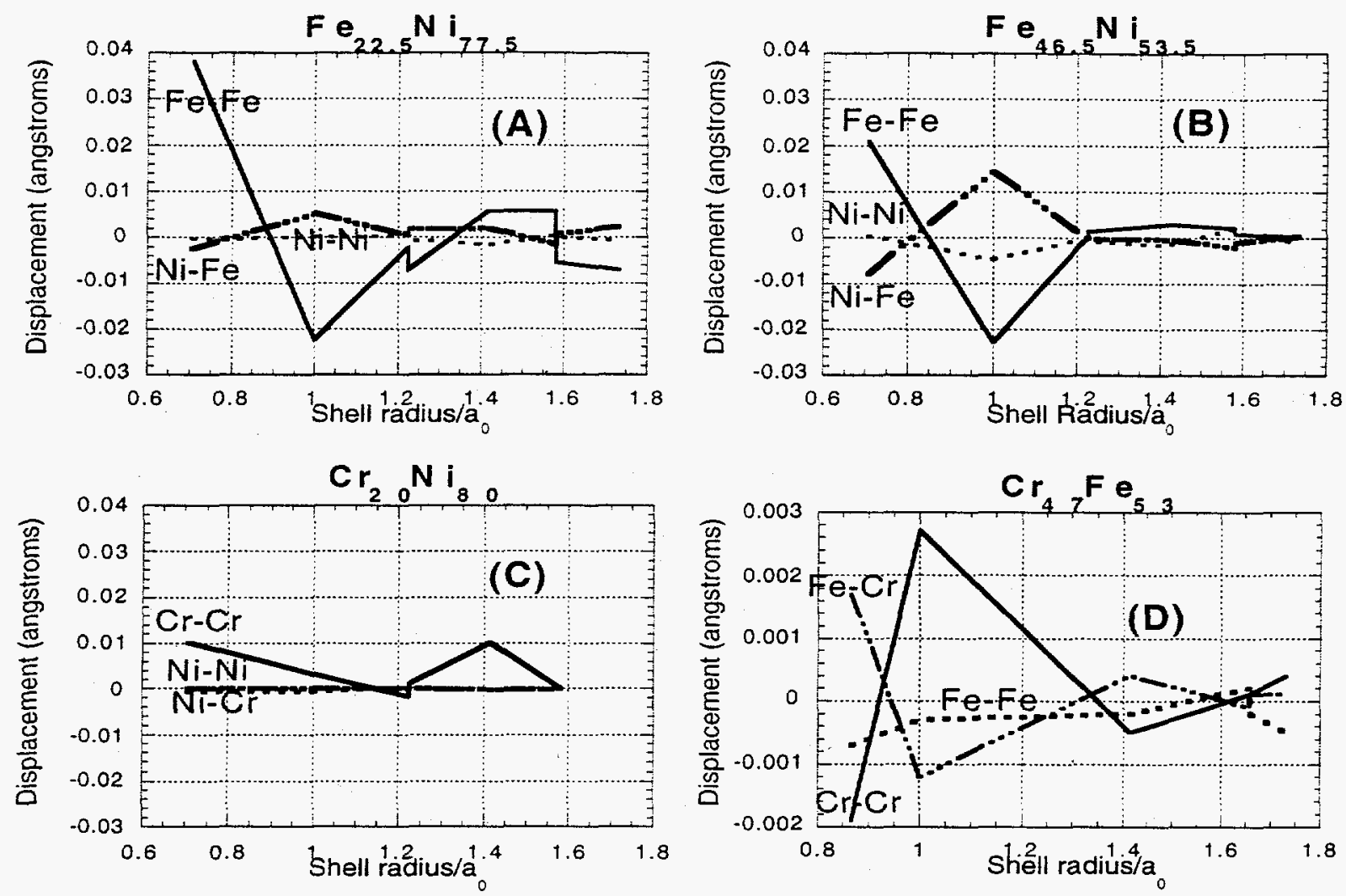

Fig. 8 Displacement from the average lattice sites for chemically specific pairs. Shell radius divided by the lattice parameter $a_{0}$ becomes 1 for second neighbors (separated by the cube edge). Data from references 2,3 , and 4.

Chakraborty $^{27}$ has proposed a compressible Ising model which can qualitatively reproduce the observed shortened nn bond distance for $\mathrm{Fe}-\mathrm{Ni}$ pairs and the expanded $\mathrm{Cr}-\mathrm{Fe}$ pairs. In this model there is a "size effect" term which reflects the different size of the atoms and an "Ising" term which is sensitive to the distance dependent Ising interaction. For systems such as the $\mathrm{Fe} / \mathrm{Ni} / \mathrm{Cr}$ binary alloys where the "size" of the atoms is similar, the unlike pair displacements do not necessarily lie between the observed like-atom displacements. Small cluster calculations appear to be another useful tool for studying the mechanisms behind the observed displacements. Of great interest is the relationship between local strains and overall lattice spacing. Recent calculations on small 12-18 atom clusters reproduce some of the observed displacement trends in Fe-Ni alloys, but the results are complicated by large surface and concentration effects ${ }^{29}$. An imbedded cluster calculation could eliminate surface effects and lead to a more complete understanding of the forces driving the observed static displacements. For a recent review of the information recovered from diffuse scattering measurements and its role in testing theoretical concepts see reference ${ }^{30}$. 


\section{$\underline{\text { V. Conclusions }}$}

The displacements of the atoms from the sites of the average lattice can be recovered from diffuse $x$-ray scattering measurements on single crystals. These measured displacements include information on the dynamic and static displacements and on the chemicallysensitive local bond lengths. The expectations of the vector displacements are recovered, including the radial and unique to these measurements the tangential displacement components. Analysis of the statistical and systematic errors show that the value of these static displacements are statistically significant. Such measurements provide new information on the real structure of crystalline solid solutions for comparison with theoretical modeling.

\section{Acknowledgments}

Our gratitude to Eliot Specht and Jin-Seok Chung for their comments on this manuscript. $X$. Jiang is supported through Sandia National Laboratory by a New Initiative from the U.S. Department of Energy, Office of Basic Energy Sciences, Division of Materials Sciences under Contract No. De-AC04-94AL85000. This research was performed in part at the ORNL/MRS beamline X-14 at the National Synchrotron Light Source, Brookhaven National Laboratory, sponsored by the Division of Materials Sciences and Division of Chemical Sciences, U.S. Department of Energy under Contract No. DE-AC05-

960R22464 with Lockheed Martin Energy Research Corporation.

\section{$\underline{\text { References }}$}

1. G. E. Ice, C. J. Sparks, A. Habenschuss, and L. B. Shaffer, Phys. Rev. Lett. 68 (1992) 863.

2. L. Reinhard, J. L. Robertson, S. C. Moss, G. E. Ice, P. Zschack, and C. J. Sparks, Phys. Rev. B 45 (1992) 2662.

3. B. Schönfeld, G. E. Ice, C. J. Sparks, H. G. Haubold, W. Schweika, and L. B. Shaffer, Phys. Status Solidi B 183 (1994) 79.

4. X. Jiang, G. E. Ice, C. J. Sparks, L. Robertson, and P. Zschack, Phys. Rev. B 57 (1996) 3211.

5. L. Vegard, Z. Kristallogr. 67 (1928) 239.

6. C. J. Sparks, G. E. Ice, X. Jiang, and P. Zschack, Mater. Res. Soc. Symp. Proc. 375 (1995) 213.

7. G. E. Ice, C. J. Sparks, J. L. Robertson, J. E. Epperson, and X. Jiang, Mater. Res. Soc. Symp. Proc. 437 (1996) 181.

8. F. Laves, Crystal Structure and Atomic Size in Theory of Alloy Phases, (American Society for Metals, Cleveland, OH; 1956) 124.

9. R. L. Fleischer, Acta Metall. 11 (1963) 203.

10. W. Hume-Rothery, The Structure of Metals and Alloys, (Institute of Metals, London, 1963). 
11. A. Zunger, in Statics and Dynamics of Alloys Phase Transitions, Vol. B319 of NATO ASI Series B, ed. P. E. A. Turchi and A. Gonis (Kluwer, Dordreht 1994) 361.

12. U. Scheuer and B. Lengeelr, Phys. Rev. B 44. 9883 (1991); G. Renaud, N. Motta, F. Lançon, and M. Belakhovsky, Phys. Rev. B 38 (1988) 5944.

13. B. E. Warren, B. L. Averback, and B. W. Roberts, J. Appl. Phys. 22 (1951) 1493.

14. B. Borie and C. J. Sparks, Acta Crystallogr. Sect. A 17 (1971) 198.

15. G. E. Ice, C. J. Sparks, and L. Shaffer, in Resonant Anomalous X-Ray Scattering: Theory and Experiment, ed. G. Materlik, C. J. Sparks, and K. Fischer (NorthHolland, Amsterdam, 1994) 265.

16. B. E. Warren, X-Ray Diffraction, (Dover, New York, 1969).

17. C. B. Walker and D. T. Keating, Acta Crystallogr. 14 (1961) 1170.

18. F. H. Herbstein, B. S. Borie, Jr., and B. L. Averbach, Acta Crystallogr. 9 (1956) 466.

19. C. J. Sparks, G. E. Ice, L. B. Shaffer, and J. L. Robertson, in Metallic Allovs: Experimental and Theoretical Perspectives, ed. J. S. Faulkner and R. G. Jordan (Kluwer Academic Publishers, Dordrecht 1994), NATO Vol. 256, 73.

20. X. Jiang, G. E. Ice, C. J. Sparks, and P. Zschack, in "Applications of Synchrotron Radiation Techniques to Materials Science," Mater. Res. Soc. Symp. Proc. 375 (1995) 267.

21. X. Jiang, J. L. Robertson, G. E. Ice, and C. J. Sparks (to be published).

22. C. J. Sparks and B. Borie, Local Atomic Arrangements Studied by X-Ray Diffraction, ed. J. B. Cohn and J. E. Hilliard (Metallurgical Society Conferences, Gordon and Breach, New York, 1965) Vol. 36, 5.

23. C. J. Sparks, Phys. Rev. Lett. 33 (1974) 262.

24. J. L. Robertson, G. E. Ice, C. J. Sparks, X. Jiang, P. Zschack, F. Bley, S. Lefebvre, and M. Bessiere (to be published).

25. S. Froyen and C. Herring, J. Appl. Phys. 52 (1981) 7165.

26. N. Mousseau and M. F. Thorpe, Phys. Rev. B 45 (1992) 2015.

27. B. Charkraborty, Europhys. Lett. 30 (1995) 531.

28. G. E. Ice, G. S. Painter, L. Shaffer, and C. J. Sparks, Nanostructural Mater. 7(12), (1996) 147.

29. W. Schweika in Statics and Dynamics of Alloy Phase Transformations, ed. P. E. A. Turchi and A. Gonis (Plenum Press, New York 1994) 103. 